\title{
I SISTEMI PER LA MISURA E LA DISPENSA DELLE ACQUE NEL DUCATO DI MILANO
}

\author{
MARIO DI FIDIO (*)
}

SUNTO. - L'autore presenta sotto un duplice profilo (storico e tecnico - scientifico) i metodi adottati per la misura e la dispensa delle acque nel territorio milanese, il quale a partire dalla fine del $12^{\circ}$ secolo è all'avanguardia in Europa nello sviluppo dell'irrigazione, mediante la costruzione di grandi canali (Naviglio Grande, Muzza, ecc.) e capillari reti irrigue, per iniziativa dei liberi Comuni. Queste reti rivelano elementi tecnici di continuità con la tradizione dei grandi acquedotti, che rifornivano l'antica Roma, distribuendo le acque ad una pluralità di utenti, fra loro in competizione. Si richiama anche l'attenzione sugli scritti idraulici di Leonardo da Vinci (fine $15^{\circ}$ secolo), il quale utilizza la tipica terminologia tecnica degli ingegneri milanesi, con cui collabora, evidenziando così il suo tributo alla tradizione idraulica locale. Un lungo processo storico di esperienze empiriche viene coronato nella seconda metà del $16^{\circ}$ secolo, con la codifica, da parte delle autorità milanesi, degli "edifici magistrali", che consentono di misurare l'acqua in modo abbastanza preciso, come gli altri beni economici, in anticipo rispetto alla rivoluzione scientifica galileiana. Questo modello empirico conserverà a lungo il primato, anche dopo la nascita dell'idraulica come scienza (B. Castelli, 1628) e sarà oggetto di ammirazione e imitazione in Europa fino alla metà del $19^{\circ}$ secolo, come testimoniano alcuni trattati francesi ed inglesi, dedicati all'irrigazione italiana. L'autore riassume anche il dibattito riformista sulla misura e la dispensa delle acque, avviato in Lombardia nell'epoca dei lumi e proseguito durante il Regno napoleonico e la Restaurazione.

$* * *$

ABSTRACT. - Adopting an historical-scientific approach, the following paper describes the methods developed during centuries for the measurement and distribution of water in the territory of Milan that, since the end of XII century, has covered one predominant role in Europe thanks to the development of advanced irrigation technologies, the construction of large canals (e.g. Naviglio Grande, Muzza) and cre-

(*) Collaboratore dell'Università degli Studi di Milano e della Beic (Biblioteca Europea d'Informazione e Cultura), Italia.E-mail: mario.difidio@fastwebnet.it 
ation of one capillary irrigation waterway network, as result of the initiative of local free institutions. This waterway network reveal technical elements from the engineering tradition of large aqueducts of ancient Rome, designed to reach a high number of final users that were in competition. A special focus is dedicated to the hydraulic writings of Leonardo da Vinci (late XV century), that uses the characteristic technical language of Milan engineers, with whom Leonardo collaborated, highlighting his tribute to the local hydraulic tradition. The long historical process, mainly based on empirical trials, finishes in the second half of the XVI century with the creation of the "Edifici Magistrali" by authorities of Milan. In advance of the Galilean scientific revolution, these special buildings allow to measure the water amount in a semi-precise way, transforming water like the other economic goods. This empirical model has maintained one leading position even after the birth of hydraulic science in 1628 (B. Castelli) and it has been admired and copied in many European countries until the mid of XIX century, as remarked in some French and English essays, devoted to Italian irrigation. The paper summarizes the reformist debate on the measurement and distribution of water amount started in Lombardy area at the time of the Enlightenment and continued during the Napoleonic Kingdom and the Restoration period.

Per secoli, il Ducato di Milano ha avuto sistemi per la misura e la dispensa delle acque all'avanguardia in Europa; essi contribuiscono a uno straordinario sviluppo dell'agricoltura, dell'economia e della società nel territorio fra il Ticino e l'Oglio, comprendente Milano, Pavia, Lodi e Cremona. Dopo la perdita dell'indipendenza, nei secoli del dominio spagnolo e poi austriaco, il Ducato si riduce a un'entità amministrativa, fino alla rivoluzione francese, quando si scioglie nel nuovo Stato creato da Napoleone nell'Italia settentrionale, dove in realtà riacquista una centralità politica, perché Milano diventa capitale. E' questo il periodo in cui l'attenzione si concentra sull'eccellenza delle pratiche irrigue milanesi, nella prospettiva della loro estensione al resto del territorio.

Questa storia s'intreccia in modo affascinante con quella della tecnica e della scienza idraulica italiane. Ne presenteremo i principali aspetti, rinviando per gli approfondimenti ad una monografia, in corso di pubblicazione presso la BEIC (Biblioteca Europea d'Informazione e Cultura), dal titolo "Idrometria", una delle prime programmate nell'ambito della "Biblioteca Idraulica Italiana", dedicata ad una collana di circa 800 libri digitali, con la collaborazione dell'Università degli Studi di Milano (Fig. 1). 


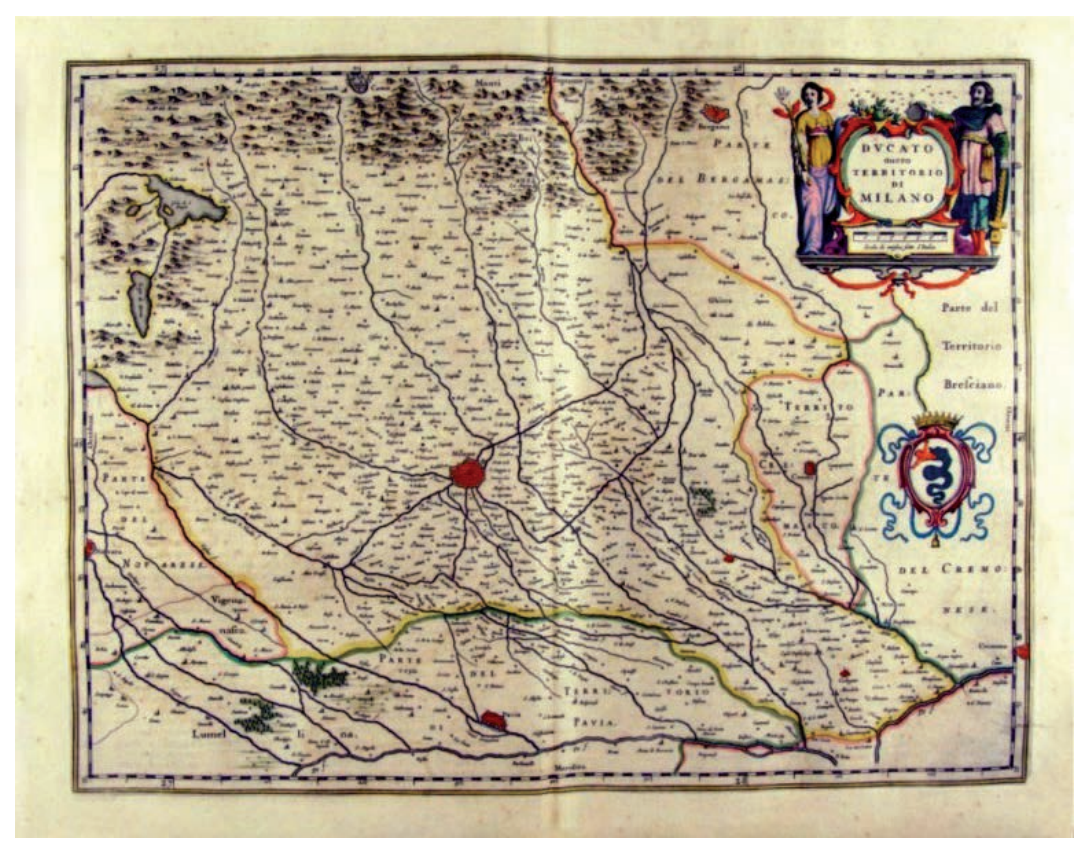

Fig. 1. Mappa del Ducato di Milano nel 1650.

\section{UNA TRADIZIONE IDRAULICA PRESCIENTIFICA ALLA BASE DI UN GRANDE SVILUPPO CIVILE}

Sono l'economia e la tecnica, non la scienza, ad aver posto per prime il problema di misurare le acque come gli altri beni, anche se oggi facciamo fatica a capirlo, perché viviamo in un mondo dominato dalla scienza, di cui la tecnica è ancella e abbiamo dimenticato il lungo periodo in cui la tecnica ha dovuto cavarsela da sola, riuscendo comunque a fare grandi cose.

Ci introduce direttamente in questo tema la lettura del trattato sulla misura delle acque, scritto nel 1628 dal bresciano Benedetto Castelli, allievo di Galileo, che segna la nascita dell'idraulica come scienza, partendo dalla definizione del concetto di portata come prodotto della sezione per la velocità e quindi del volume nell'unità di tempo. Divertente è l'aneddoto da lui riferito sull'architetto romano Giovanni Fontana, il quale, dopo la piena del Tevere nel 1598, calcolava la portata di tutti gli immissari del fiume sommando semplicemente le sezioni e concludeva che, per 
una difesa efficace di Roma dalle inondazioni, era necessario costruire almeno due altri alvei artificiali, uguali a quello naturale del Tevere!

Ovviamente si ride, ma stiamo attenti a non ridere troppo. Riflettiamo su un paradosso illuminante: la Roma dei Cesari e dei Papi, da Frontino a Fontana, pur ignorando la scienza idraulica moderna, ha sempre bevuto l'ottima acqua sorgiva dei suoi grandi acquedotti, mentre Parigi ha bevuto l'acqua della Senna fino a Napoleone III e al barone Haussmann, oltre due secoli dopo la rivoluzione scientifica!

E' logico che Castelli affermasse in modo polemico le ragioni della rivoluzione scientifica avviata dal suo maestro Galileo: occorreva uscire dal mondo del pressappoco per entrare in quello della precisione. Ma oggi siamo chiamati a valutare in modo più equanime proprio il mondo del pressappoco, ossia quello della tecnica pregalileiana. Se avevano cognizioni così rozze, non ci si spiega come abbiano fatto gli antichi Romani a costruire grandi acquedotti e i Milanesi a costruire grandi canali navigabili ed irrigui.

In Italia la questione è complessa, proprio perché la rivoluzione scientifica inizia proprio qui, con una straordinaria fioritura nel Seicento: dopo Castelli, altri personaggi come Torricelli, Cabeo, Barattieri, Michelini, Montanari, Guglielmini sviluppano la scienza idraulica moderna, creando quella che verrà chiamata la scuola idraulica italiana. Si afferma una nuova figura: il fisico - matematico (il "geometra", come allora si diceva), in opposizione alle "pratiche volgari" di "architetti ed ingegneri d'acque"; ma dopo gli attriti iniziali le due figure trovano una nuova sintesi, ossia il geometra diventa anche ingegnere d'acque e viceversa (come è già evidente in Barattieri).

Partire da questa storia, che pure onora grandemente la cultura italiana, ignorando quella precedente sarebbe un errore: l'Italia ha anche un primato idraulico prescientifico, perché nel Medioevo costruisce, in tutte le regioni settentrionali, una rete di canali navigabili ed irrigui; nel Rinascimento il Ducato di Milano, dopo esperienze plurisecolari sulle bocche d'erogazione delle acque, mette a punto edifici detti magistrali, che consentono di raggiungere risultati di notevole precisione (considerati i limiti di conoscenza del tempo) per la misura e la dispensa delle acque, senza bisogno di fare calcoli.

Ancor più stupisce che in Lombardia siano stati costruiti moltissimi canali, grandi e piccoli, a partire dalla fine del $12^{\circ}$ secolo. Com'è stato possibile? La spiegazione più logica appare il collegamento con la tradizione romana. I Romani antichi non hanno costruito soltanto gran- 
di acquedotti, ma anche canali navigabili ed irrigui, di cui abbiamo alcune tracce in Lombardia, nel Canale della Muzza, nome derivato dai coloni della gens Mutia e nel Naviglio grande bresciano.

A seguito delle invasioni barbariche questa grande tradizione costruttiva sembra totalmente dimenticata, ma non è così, perché subito dopo la battaglia di Legnano e la pace di Costanza (1183) tra i Comuni lombardi e il Sacro Romano Impero, che cede loro i diritti sulle acque pubbliche (con le parole Jura fluviorum illi cuique populo Caesares servante), si apre una stagione straordinaria di opere idrauliche. Due grandi canali, quasi novelli fiumi, diventano i prototipi di una nuova storia della navigazione interna e dell'irrigazione europea: il Naviglio Grande, che collega Milano al Ticino ed al Lago Maggiore ed è nel contempo navigabile ed irriguo; il Canale Muzza, con finalità unicamente irrigue, derivato dall'Adda nel Milanese fino a Paullo e poi nel Lodigiano (Fig. 2). Elia Lombardini (1844) afferma la continuità di questa tradizione costruttiva mediante un trasferimento ininterrotto di conoscenze tecniche dall'epoca romana: "La vera cagione che mosse ad intraprendere quasi contemporaneamente opere cosi grandiose si vede ben piuttosto nel generale risurgimento di tutta l'antica civiltà, nella novella potenza dei municipj dopo la pace di Costanza, nella vicendevole emulazione delle cittadinanze, la quale si spingeva ad altre ben maggiori estremità, e nell' ingegno costruttivo di questi popoli, i quali dalla non interrotta tradizione delle opere e delle consuetudini precedenti avevano già le più prossime tracce di simili imprese". In ogni caso, gli ingegneri idraulici lombardi del Medioevo applicano con sicurezza queste tecniche in patria e le esportano in tutta Europa.

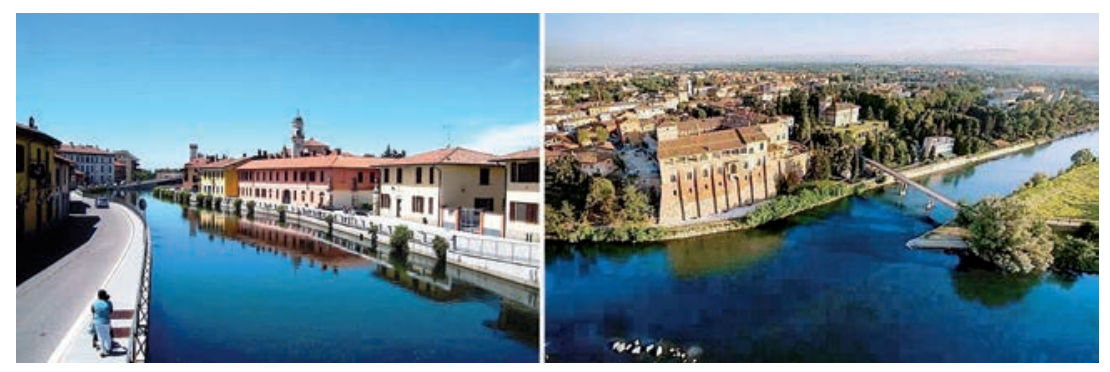

Fig. 2. A sinistra il Naviglio Grande a Gaggiano. A destra l'incile della Muzza, derivata dall'Adda a Cassano d'Adda sotto la Rocca Viscontea. La creazione di questi due grandi canali nel territorio milanese e lodigiano, rispettivamente alla fine del XII $e$ all'inizio del XIII secolo, è il fatto capitale nella storia della navigazione interna e dell'irrigazione europea. 
Dal Duecento, nel Milanese entra un volume enorme di acque, derivate dal Ticino e dall'Adda, distribuite capillarmente nel territorio ed utilizzate non solo per l'irrigazione, ma per una molteplicità di usi artigianali e industriali grazie alle ruote d'acqua. Questo, assieme ai vantaggi commerciali della navigazione da Milano al Lago Maggiore, è il principale fattore dello sviluppo economico e civile di Milano. All'epoca di Ludovico il Moro, il Ducato di Milano raggiunge una prosperità straordinaria, che si riflette nel lusso di palazzi, carrozze, vestiti e sorprende il visitatore, pur abituato allo splendore di tante città italiane.

\section{LEONARDO E IL BATTENTE IDRAULICO}

Nell'ultimo ventennio del Quattrocento, alla corte di Ludovico il Moro si trova Leonardo da Vinci, che è considerato il precursore della scienza idraulica. Nei suoi codici troviamo dispersi i contenuti di un vero e proprio trattato d'idraulica (superiore a quello di Castelli!), che nel Seicento un oscuro monaco milanese, figlio naturale del conte Arconati, ricompone pazientemente; il testo (redatto nel 1643) resta sepolto nella Biblioteca Barberini a Roma, per essere pubblicato soltanto nel 1826 a Bologna: troppo tardi per influire sullo sviluppo scientifico.

Quest'affascinante storia milanese, ricca di mistero, suscita polemiche, innescate da un'improbabile ipotesi di plagio di Leonardo da parte di Castelli, avanzata da Elia Lombardini nel 1860. Ma in questa sede del trattato leonardesco ci interessa soprattutto la parte che si riferisce all'efflusso dalle luci e quindi alla misura e dispensa delle acque.

Il primo elemento che attira l'attenzione è di natura filologica (vedi Di Fidio - Gandolfi, 2013). Leonardo è un toscano che, nel lungo soggiorno milanese, contamina la sua lingua nativa con quella degli ingegneri locali, usando termini specialistici presenti solo a Milano. Per esempio il canale minore di derivazione non è "gora" alla toscana, ma "rugia" = "roggia" alla milanese; è il percorso opposto a quello del Manzoni, il quale, dopo aver sciacquato i panni in Arno, ribattezza con "gora" il fossato che circonda il Lazzaretto di Milano. Analogamente, Leonardo usa più volte il termine "battente", che indica la distanza tra il punto più alto di una luce o bocca, aperta nella parete di un recipiente, e il pelo libero dell'acqua del recipiente stesso, da cui la bocca attinge (per es: "Se la bocca fosse due once ed il battente due, e tu alzassi la bocca di un'oncia, allora tu diminuisci la metà della potenza a esso battente"). 
Si consideri che, due secoli dopo, nei trattati idraulici del Seicento (con l'eccezione di quello di Barattieri, che lavora nel Ducato di Milano!), il termine battente, oggi di uso comune in Italia (ma non all'estero!), è ancora ignorato; per indicare questa grandezza fisica si usano faticose perifrasi (v. per es. Guglielmini, 1697). La presenza del battente negli scritti di Leonardo testimonia che, nel Ducato di Milano, la misura delle acque è più evoluta, almeno dalla seconda metà del Quattrocento (quindi prima della codifica degli edifici magistrali!), perché introduce un parametro fondamentale per determinare la velocità d'efflusso. Inoltre il battente è indicato da Leonardo nella misura di due once lineari, che coincide con quella dell'edificio magistrale milanese, così come codificato nella seconda metà del Cinquecento (Fig. 3).

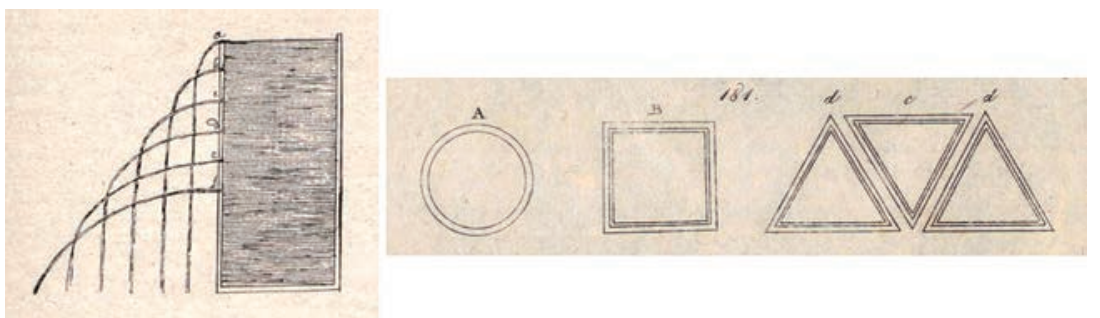

Fig. 3. Leonardo da Vinci, verso la fine del Quattrocento, studia le bocche irrigue nel Ducato di Milano. La figura a sinistra illustra l'efflusso dalle luci ("quantità dell'oncia dell'acqua secondo l'altezza o bassezza della superficie"), che prende forza col crescere del battente. La figura a destra presenta diverse bocche irrigue ("delle bocche equali e d'equal altezza, quella versa più acqua in pari tempo, che averà maggior somma di sé nella sua parte inferiore, che nella parte di sopra").

Leonardo è attentissimo alle modalità di misura e dispensa delle acque estratte dalle bocche dei canali irrigui. Non gli sfugge la complessità delle situazioni reali, spesso profondamente diverse dai modelli teorici. Per la vera misura dell'oncia dell'acqua (un altro termine milanese!), egli distingue ben sedici modalità (per es. altezza e velocità dell'acqua; obliquità, spessore e figura della bocca, ecc.), fornendo per ciascuna di esse dettagliate indicazioni pratiche. Si rifletta che Leonardo studia bocche, come quelle del Naviglio Grande, esistenti da tre secoli; una domanda sorge spontanea: quante di queste considerazioni sono il frutto di osservazioni originali del grande genio toscano e quante invece sono dedotte dal patrimonio di conoscenze pratiche degli ingegneri 
milanesi, lentamente sedimentate nei secoli, in cui essi hanno provato e riprovato, sbagliato e corretto nella disposizione delle bocche?

Sorprendente è il confronto fra il testo di Leonardo e quello di Frontino (De aquaeductibus urbis Romae), uscito attorno al $100 \mathrm{~d}$. C. sotto Traiano e commentato dal marchese Poleni nel 1722. Come curatore delle acque, Frontino è molto interessato al tema dell'idrometria, perché vuole scoprire le malefatte dei fontanieri, che rubano spudoratamente l'acqua pubblica. E' vero che egli misura le quantità d'acqua in base alla sezione di canali e condotti, ma sceglie le sezioni di controllo in modo che la velocità non sia né troppo grande né troppo piccola (in medio stat virtus!), omologando in tal modo le varie misure; inoltre prescrive che le bocche di consegna abbiano tutte la stessa altezza e disposizione (quindi lo stesso battente e la stessa velocità!). Evidentemente si è posto il problema di misurare l'acqua corrente con più esattezza.

Curiosamente, un disegno di Leonardo sulle bocche dei navigli milanesi ne richiama uno di Frontino (ricostruito da Poleni), sulle bocche degli acquedotti romani. In entrambi i casi si mostrano tre bocche di diametro uguale e alla stessa altezza, alimentate dal medesimo canale; la quantità d'acqua dispensata è progressivamente crescente in funzione della disposizione delle bocche rispetto alla corrente (Fig. 4). Frontino (art. 36) sceglie la bocca intermedia, scartando le altre due: tutti i calici dovranno essere ortogonali rispetto alla corrente ed orizzontali (in rectum et ad libram collocati) alla stessa quota; è il principio della formazione dei moduli (il termine modulus è usatissimo nel suo trattato, in senso moderno!). E' chiaro che Frontino, per formazione un militare, ha dedotto queste conoscenze dai fontanieri ai suoi ordini. Si può dunque constatare che fra i due corpi tecnici, preposti rispettivamente agli acquedotti romani e ai navigli milanesi, a distanza di più di un millennio c'è un'affinità del modus operandi, attraverso secoli di esperienze empiriche che sedimentano una notevole sapienza tecnica e creano un comune humus culturale.

Ma Leonardo è uno scienziato che va oltre le modalità pratiche di costruzione delle bocche, perché si pone il problema di scoprire il rapporto esatto tra la velocità d'efflusso ed il carico idraulico sulla luce (collegato al battente), anche se sbaglia affermando la proporzionalità fra queste due grandezze fisiche, come in seguito farà anche Castelli. Il primo a scoprire, con l'esperienza e il ragionamento, che la velocità è proporzionale alla radice quadrata del carico, sarà Evangelista Torricelli (1644); la sua è una legge fondamentale per lo sviluppo successivo della 
scienza idraulica fisico - matematica, fino all'idrodinamica dei liquidi perfetti di Daniele Bernoulli (1738).

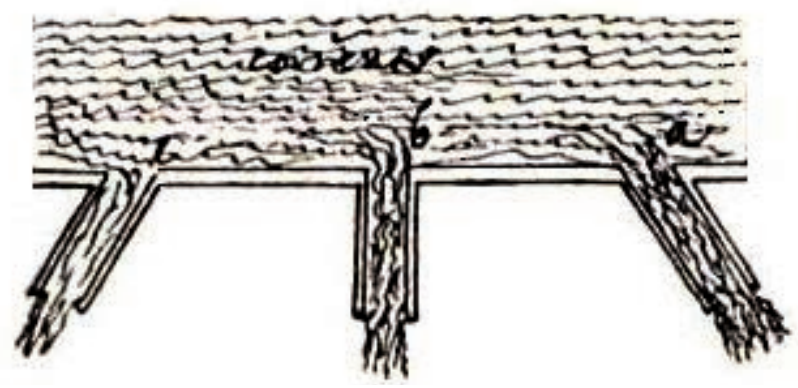

Fig. 4. Le modalità per la misura dell'oncia d'acqua in Leonardo da Vinci (circa 1500):

"Delle bocche eguali e simili, quella verserà più acqua, che haverà li suoi lati più obliqui verso l'avvenimento della corrente del canale; e di converso quella verserà men'acqua, che haverà li lati più obliqui inverso la fuga della medesima corrente, cioè verserà più acqua la bocca $\mathrm{A}$, che le bocche $\mathrm{B} \mathrm{e} C$ ”. Delle tre bocche $C, B, A$, investite progressivamente dalla corrente, la bocca $A$ versa più acqua di $B$ e $B$ di $C$. Le medesime considerazioni si trovano in Frontino (100 d.C.), secondo cui tutte le bocche devono essere come quella mediana (in medio stat virtus).

Ovviamente gli ingegneri idraulici lombardi del Rinascimento non potevano saperne più di Leonardo. Ma, dal loro punto di vista, era sufficiente sapere che il battente, assieme alla sezione della bocca, era il parametro determinante per la portata d'efflusso, senza la necessità di conoscere con esattezza la legge che collega queste grandezze fisiche. La genialità della tecnica idraulica lombarda sta appunto nell'aver aggirato il problema, codificando un manufatto che garantiva - in misura accettabile l'esattezza dell' "oncia d'acqua" erogata all'utente, senza eseguire calcoli, all'inizio impossibili, nell'ignoranza della legge fisica dell'efflusso, e dopo Torricelli, per un lungo periodo, laboriosi ed aleatori.

Si consideri che, dopo la scoperta di Torricelli, per secoli in tutta Europa numerosi scienziati, detti Foronomisti (tra cui in Francia Mariotte, in Inghilterra Newton e in Italia Poleni), si dedicano con passione al perfezionamento della formula da lui scoperta, attraverso esperienze per quantificare molteplici effetti, che influiscono sul calcolo esatto della portata: la contrazione della vena liquida effluente, lo spessore della luce (in parete grossa o viceversa sottile), la presenza di rigurgito parziale o totale a valle, la variazione del carico idraulico sui singoli punti delle grandi luci, ecc. Mentre la scienza idraulica faticosamente 
avanzava nel suo cammino, l'uso di formule per il calcolo diretto delle portate d'efflusso dalle luci da una parte superava le modeste capacità degli operatori rurali (i campari), dall'altra non sembrava o non era abbastanza affidabile o infine confliggeva con i diritti acquisiti. Non sorprende quindi che, per un periodo assai lungo oltre la rivoluzione scientifica, fino alla prima metà dell'Ottocento, gli edifici magistrali, grazie ai loro requisiti di affidabilità, abbiano continuato ad essere apprezzati da molte generazioni d'ingegneri idraulici, ormai aggiornati sui progressi della scienza.

\section{IL SISTEMA MEDIOEVALE DELL'ONCIA D'ACQUA: UNA PORTATA VIRTUALE}

Per comprendere una situazione così complessa e lontana nel tempo, si deve spiegare il significato della misteriosa "oncia d'acqua". Fino all'introduzione del sistema metrico decimale da parte di Napoleone, in Italia (come nel resto d'Europa), il sistema di misura è quello in dodicesimi ereditato da Roma, con l'aggravante che la frantumazione politica nel Medioevo ha moltiplicato le unità di misura (piede o braccio) in uso sul territorio, anche all'interno dei successivi, più vasti Stati rinascimentali; così nel Ducato di Milano il piede milanese è diverso dal lodigiano e questo dal cremonese, ecc. In ogni caso, tale sistema consente di calcolare lunghezze, superfici, volumi e pesi per tutte le merci, ma non per l'acqua, la quale fa eccezione, perché è misurata in unità particolari, le quali ricevono a loro volta nomi diversi: oncia d'acqua nel Ducato di Milano, quadretto nella Repubblica veneta, ruota d'acqua in Piemonte, ecc.

Che cosa s'intende esattamente per oncia d'acqua? Non si tratta di una quantità precisa, erogata nell'unità di tempo (per esempio una $\mathrm{o}$ più once cubiche il minuto, essendo l'oncia lineare l'unità di misura locale), bensì di una portata virtuale: è la quantità d'acqua che esce in un dato tempo da una bocca (il modulo locale) di forma e dimensioni date (misurate in once lineari ed eventualmente con altre caratteristiche prescritte, per es. nel Milanese il "battente") per garantire che i volumi erogati siano multipli o sottomultipli fedeli del modulo locale.

L'acqua, bene di così grande importanza per l'uomo, è dunque l'unico che non possa essere misurato con esattezza: chi compra un'oncia d'acqua non sa con precisione quante once cubiche gli saranno ero- 
gate nel corso del tempo. Il sistema dell'oncia d'acqua continua fino all'Ottocento, addirittura dopo l'unificazione delle misure con il sistema metrico decimale, sia pure in forme gradualmente residuali (vedasi Turazza, 1867). Le cause di questa situazione sono da ricercarsi nel sistema giuridico che perpetua gli antichi diritti espressi in once d'acqua e nel tenace conservatorismo del mondo rurale, diffidente nei confronti delle riforme. Nella fase finale questi fattori sono più forti di quelli tecnici e scientifici.

La valutazione storica dell'oncia d'acqua non può peraltro esaurirsi in queste considerazioni critiche: sarebbe un giudizio troppo sbrigativo ed anacronistico. Entrando più a fondo in questo sistema, ormai così estraneo alla nostra mentalità, si scopre che anch'esso è suscettibile di evoluzione tecnica e scientifica ed è precisamente quello che è avvenuto nel corso dei secoli. Il panorama che si presenta ai nostri occhi è stupefacente e affascinante per vari motivi: l'estrema variabilità territoriale dei sistemi di misura, la convivenza, anche a breve distanza, di sistemi evoluti e di altri molto arretrati, che non fanno alcun conto della velocità ignorando la lezione di Castelli ed infine la presenza, ai vertici, d'alcuni modelli precastelliani, che possono essere definiti d'eccellenza, ossia gli edifici magistrali del Ducato di Milano.

La situazione è complessa anche perché, a partire da Castelli (1628), che definisce il concetto di portata reale (per es. in piedi cubici al minuto), ingegneri e periti d'acque incominciano a calcolarla, o meglio (nell'ignoranza dei coefficienti di proporzionalità) all'inizio calcolano i rapporti tra le portate reali di due sezioni a confronto: se si tratta di bocche, applicano la formula di Torricelli; se si tratta di corsi d'acqua, applicano la formula del moto uniforme di Castelli o quella di Guglielmini, entrambe inesatte, perché ipotizzano un rapporto lineare, e rispettivamente parabolico, tra velocità media ed altezza delle corrente (Fig. 5).

Se i detti sistemi di calcolo sono tutti imperfetti, per scoprire le portate dei corsi d'acqua, inclusi i canali rurali, dal Seicento l'Italia sviluppa un sistema d'eccellenza, basato su misure dirette di velocità puntuali o medie lungo le verticali, attraverso una molteplicità di tachimetri idraulici, continuamente perfezionati, fino ai primi decenni dell'Ottocento. Queste misure - ovviamente riservate ad ingegneri e periti d'acque - consentono di disegnare il solido di portata, giungendo così a calcoli delle portate reali più precisi di quelli delle formule. Insomma, per alcuni secoli, l'oncia d'acqua e gli edifici magistrali con- 
vivono con sistemi più moderni per la misura delle acque; in particolare gli idraulici italiani hanno gli strumenti per verificare l'attendibilità degli edifici magistrali, confrontando i rapporti teorici fra le once d'acqua con $\mathrm{i}$ rapporti reali fra le portate erogate.
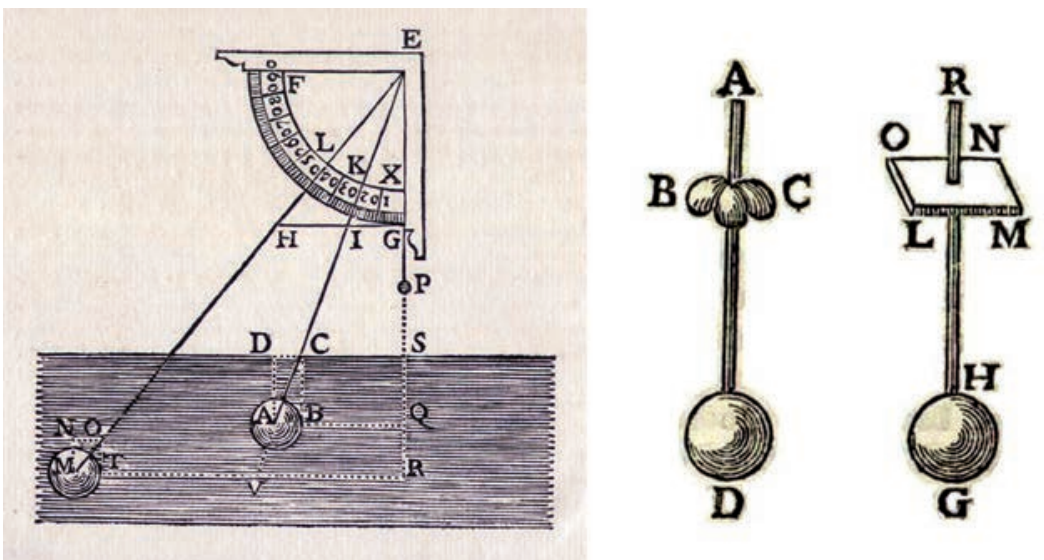

Fig. 5. A sinistra, la palla a pendolo di Guglielmini (1690) per la misura della velocità puntuale nelle sezioni dei corsi d'acqua.

A destra, l'asta idrometrica di Cabeo (1646) - Barattieri (1663), per la misura della velocità media lungo determinate verticali (figure tratte da Lecchi, 1765).

Questi ed altri numerosi tachimetri idraulici inventati dagli Italiani consentono di misurare con precisione la portata reale dei corsi d'acqua, inclusi i canali rurali.

\section{GLI EDIFICI MAGISTRALI, MODULI A BATTENTE COSTANTE}

Conosciamo la regolazione dell'efflusso dalle luci negli edifici magistrali del Ducato di Milano (milanese, cremonese, lodigiano, ecc.), attraverso gli atti di codifica, risalenti al Cinquecento, presentati da Giuseppe Bruschetti nella sua Storia dei progetti e delle opere per l'irrigazione del Milanese (1834) e i commenti in vari scritti di idraulici italiani (Barattieri 1663, Lecchi 1765, Frisi 1777, Ferrari 1779, Brunacci 1814, Tadini 1816, Venturoli 1818, Possenti 1858, ecc.). La prima codifica di questi manufatti avviene nel Cinquecento, da parte del Senato di Milano e precisamente nel 1551 per l'edificio magistrale cremonese e nel 1571 per quello milanese. L'ultimo tentativo di aggiornamento è quello dell'ing. Carlo Possenti (1858). 
Si è già visto che gli ingegneri rinascimentali conoscono bene l'influsso del battente, e quindi del carico idraulico, sulla portata effluente da una luce, ma non sono in grado di collegare, con una legge matematica, l'uno e l'altra, ricavando il valore assoluto della portata; neppure riescono a calcolare il rapporto tra le portate effluenti da luci con carichi diversi. Decidono allora di aggirare il problema: mediante una forma opportuna del manufatto e le manovre di una saracinesca, il battente delle luci dovrà essere costante al variare del livello nel corso d'acqua dispensatore ed anche le luci dovranno conservare l'altezza costante, variando soltanto la larghezza, in modo tale da assicurare l'eguaglianza dei carichi idraulici. Ricorrono inoltre ad opportuni artifici per assicurare, il più possibile, l'indipendenza delle luci dalle manovre degli utenti a valle. Il difetto forse più rilevante di questi manufatti è la mancata considerazione della contrazione della vena liquida effluente dalle luci, che varia con la larghezza delle stesse; invece può essere trascurabile la variabilità delle perdite di carico, dovute all'attrito contro le pareti delle luci, poiché si tratta in assoluto di grandezze modeste.

In ogni caso, gli ingegneri del Ducato di Milano riescono a definire un modulo (l'oncia d'acqua), il quale eroga una portata che è garantita abbastanza bene nella sua costanza temporale; anche se non sono in grado di determinarne il valore assoluto (come volume nell'unità di tempo), possono calcolarne facilmente i multipli e i sottomultipli variando la larghezza delle luci. In tal modo, anche l'acqua può essere misurata come un mucchio di fieno, come i mattoni da fabbrica, come un monte di terra, per dirla come Romagnosi (1823). Empiricamente, questo modulo è correlato, con discreta precisione, ai vari usi dell'acqua, per muovere le macchine idrauliche, irrigare le campagne, ecc. Non è un vantaggio da poco per l'economia e lo sviluppo civile ed esso è reso possibile grazie a metodi empirico - razionali, in largo anticipo rispetto alla rivoluzione scientifica galileiana del Seicento.

\section{UNA STUPEFACENTE DIVERSITÀ TERRITORIALE CHE CONVIVE CON UNA GRANDE CULTURA UNITARIA}

Sappiamo bene quanto l'Italia sia complessa e diversa nelle sue parti. Ciò si riflette anche sullo sviluppo storico della scienza e della tecnica idrauliche, ma in forme sorprendenti, per noi moderni, spesso con- 
dizionati dagli stereotipi dell'attualità, che riguardano sia i rapporti fra le singole regioni italiane sia i rapporti fra l'Italia nel suo complesso e gli Stati dell'Europa centrale e settentrionale, considerati come modelli.

Fino all'unità nazionale, scienza e tecnica sono coltivate soprattutto nelle regioni centrali e settentrionali, diciamo da Roma in su, e questo in parte conferma ed in parte smentisce gli stereotipi (per l'insospettata importanza dell'idraulica romana!). Più sorprendente è il fatto che anche nel Centro - Nord ci siano fortissime differenze, al punto che aree d'eccellenza per l'idraulica fluviale (come l'Emilia - Romagna e il Veneto) sono arretrate nel campo dell'irrigazione e di conseguenza nella misura e dispensa delle acque irrigue. E ciononostante tutti gli idraulici italiani dal Seicento all'inizio dell'Ottocento sentono di appartenere alla stessa, grande tradizione culturale e difendono orgogliosamente il primato della scuola idraulica italiana in Europa; le polemiche interne, sempre molto accese secondo il carattere italiano, non devono fuorviare.

Fino all'unità d'Italia soltanto a nord del Po sono presenti vaste reti di canali irrigui e sistemi evoluti per la misura e il riparto delle acque irrigue, sia pure con efficienza diversa e in prevalenza entro i limiti concettuali dell'oncia d'acqua. Nell'Italia centrale e meridionale troviamo invece grandi acquedotti civili d'eccellenza europea, sul modello degli antichi acquedotti romani. A sua volta l'Italia settentrionale presenta un quadro molto diversificato: l'area più ricca di acque è quella del Ducato di Milano, fra il Ticino e l'Oglio, che ha sviluppato i sistemi tecnologicamente più avanzati. La seconda area interessante per lo sviluppo dell'idrometria irrigua è quella mantovana - veronese, oggetto di un trattato internazionale fra la Casa d'Austria e la Repubblica di Venezia nel 1764. Le altre province (venete, emiliane e piemontesi) sono più arretrate, anche perché più povere di acque disponibili per l'irrigazione.

Il bresciano Benedetto Castelli (1628), che insegna a Roma, si esprime sul tema della dispensa delle acque avendo riguardo soprattutto al riparto negli acquedotti romani. Più interessante sotto questo profilo è il trattato del suo rivale, il ferrarese Nicolò Cabeo (1646), il quale elabora un'interessante strategia giuridica e tecnica e definisce modalità precise, ancorché laboriose, per distribuire le acque tra i singoli utenti, ossia propone di misurare direttamente i volumi nell'unità di tempo (e quindi le portate assolute!), tarando pezzo per pezzo le reti idriche dei condotti e dei canali; ma siamo nell'ambito di una teoria. 
I trattati di Domenico Guglielmini (1697) e Bernardino Zendrini (1741) forniscono un quadro della dispensa delle acque, che si sforza di razionalizzare pratiche complessivamente arretrate nelle aree dove operano, ossia il Bolognese ed il Veneto. Ma si può dire che, su questo tema, entrambi volino basso rispetto alle altezze raggiunte nell'idraulica fluviale, dove eccelle la scuola idraulica italiana.

Notizie dettagliate sulla misura e la dispensa delle acque in Piemonte si trovano in Francesco Domenico Michelotti (1767 - 1771) e rivelano una notevole arretratezza rispetto al confinante Ducato di Milano, dove da secoli si usano gli edifici magistrali. Fanno eccezione unicamente il Novarese e la Lomellina, dove è presente l'edificio magistrale; la ragione storica è evidente: queste province sono rimaste unite a Milano fino all'inizio del Settecento. Quindi il Milanese ha influito sul progresso della tecnica irrigua in Piemonte, anche se l'ambizione di Casa Savoia fa la sua parte, perché nel 1765 porta alla costruzione dello Stabilimento per le sperienze idrauliche La Parella (Torino), il primo laboratorio permanente d'idraulica in Europa.

Fino alla metà del Settecento, l'edificio magistrale appare ignorato dai grandi trattati d'idraulica, con l'eccezione di quello di Giambattista Barattieri (1556-1663) che lo conosce bene, in quanto direttore del Canale Muzza facente capo all'Ufficio camerale di Milano (Fig. 6); lo ignorano invece il bresciano Castelli (1628), il ferrarese Cabeo (1646), il bolognese Guglielmini (1690, 1697), il cremonese Grandi, che opera in Toscana (1723) e il camuno Zendrini, che opera a Venezia (1741). Emblematico è un episodio narrato da Francesco Bernardino Ferrari nella sua lettera al conte di Rogendorf (1779) responsabile dell'amministrazione demaniale dei Navigli: un ospite straniero del conte in visita a Milano è dapprima incredulo e poi ammirato di fronte al funzionamento dell'edificio magistrale milanese, che non ha eguali e si meraviglia che i Milanesi non l'abbiano da tempo fatto conoscere all'Europa; si capisce il motivo: non lo conoscono bene neanche in Italia! D'altra parte non è una novità: nei secoli, l'Italia ha sviluppato molte eccellenze e spesso le trascura.

Oltre alle ragioni in precedenza indicate, il motivo di questo scarso interesse sembra da ricondurre al fatto che il Ducato di Milano, dopo gli splendori della corte di Ludovico il Moro e di Leonardo, è caduto, per così dire, in un cono d'ombra durante il lungo dominio spagnolo. Tuttavia la figura di Barattieri, un ingegnere piacentino - lodigiano aggiornato alla scuola di Castelli, non è certamente secondaria, poiché il 
suo trattato, assieme a quello del matematico romano - toscano Famiano Michelini (Trattato della direzione de' fiumi, 1664), anticipa la maturazione dell'idraulica fluviale come scienza, che giunge al culmine con il matematico - medico bolognese Domenico Guglielmini (Della natura de' fiumi, 1697), a testimonianza di una solida ed originale cultura unitaria italiana, sotto la scorza delle divisioni politiche.

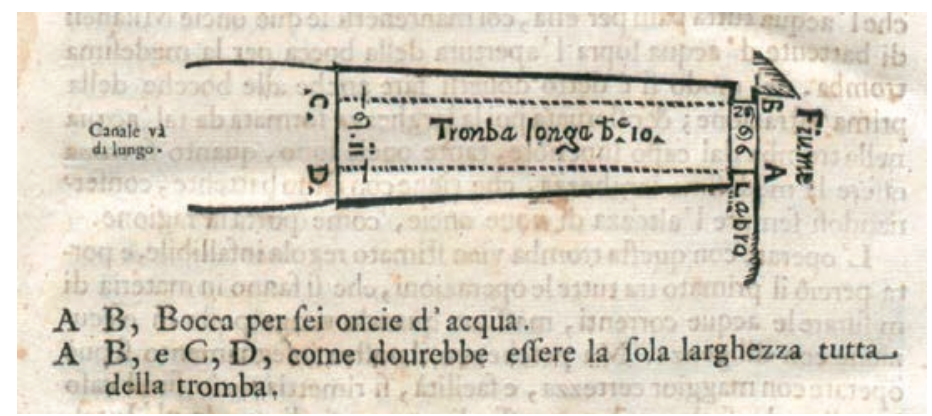

Fig. 6. Schema della tromba applicata alla bocca lodigiana verso la metà del Seicento: l'allargamento della tromba rimane fisso al variare della bocca di riferimento. Giambattista Barattieri consiglia di conservare la medesima larghezza della bocca stessa per tutta la lunghezza della tromba (da Barattieri, 1663).

Nel Settecento la comparsa sul teatro scientifico italiano, con esperienze in varie regioni, di eminenti idraulici milanesi, come Antonio Lecchi e Paolo Frisi, riporta l'attenzione sul confronto tra i diversi sistemi per la dispensa delle acque e apre un dibattito riformista che giungerà a piena maturazione nel periodo napoleonico.

\section{IL DIBATTITO RIFORMISTA SULLA MISURA E LA DISPENSA DELLE ACQUE NELL'EPOCA DEI LUMI}

Il dibattito si apre con il trattato del milanese Antonio Lecchi (1765), insegnante al collegio dei Gesuiti di Brera, che potremmo definire un riformista moderato in virtù di un notevole realismo. Egli vive nell'area più avanzata d'Europa, nell'arte dell'irrigazione e della misura delle acque e ritiene di dover dedicare le sue riflessioni soprattutto al ricupero delle situazioni più arretrate, guardandosi dalla tentazione di proporre l'estensione immediata a tutto il territorio degli edifici magistrali. A differenza della maggior parte dei trattatisti idraulici, che 
hanno una concezione eminentemente scientifica e tecnica ed appaiono quasi in imbarazzo di fronte a problemi che faticano a governare per i loro aspetti giuridici, politici, sociali ed umani, a questi ultimi Lecchi appare molto attento ed ha il merito di mettere tutte le carte in tavola, anche se la sua posizione certamente non può piacere agli scienziati illuministi a lui contemporanei, come il concittadino Paolo Frisi.

Lecchi, formalmente rispettoso della tradizione, non rinuncia ad apportare al sistema di misura delle acque gli opportuni miglioramenti, ed anzi numerosi sono i suoi suggerimenti pratici al riguardo, ma egli cerca, possibilmente, di adattarli alle leggi e tradizioni locali e tiene conto realisticamente della natura umana: i campari non sono angeli! (come non lo erano i fontanieri di Frontino!). Le soluzioni da lui suggerite, pur derivando da una solida conoscenza tecnica e scientifica, non rappresentano uno stravolgimento, ma una più corretta interpretazione delle leggi vigenti nella loro sostanza, che certamente non può giustificare l'iniquità del riparto delle acque, e nella forma consuetudinaria a cui gli utenti sono da gran tempo abituati: ai suoi occhi appaiono quindi più realistiche e fattibili, in un processo di riforma graduale, che col tempo consentirà di superare anche le forme tradizionali inadeguate.

Notevole è il suggerimento di aggirare il problema della diversità dei moduli locali d'oncia d'acqua (alcuni dei quali addirittura non prevedono il battente!) adottando, per così dire, un codice di traduzione da un sistema all'altro, ossia identificando, mediante misure dirette di portata nel canale alimentato (eseguite con i tachimetri idraulici dell'epoca, come la palla a pendolo o l'asta idrometrica), un modulo rettangolare sotto battente, che abbia la stessa portata di tale oncia formale e possa essere utilizzato successivamente come base per tutte le misure delle bocche a confronto tra loro. Sorprendentemente, ma non troppo, esistono punti di contatto tra il pensiero di Lecchi e quello di esponenti di un riformismo più radicale, come Tadini (1816) e Romagnosi (1823), altrettanto capaci di concezioni strategiche.

Lecchi esamina criticamente anche un manufatto di misura più semplice dei moduli, assai diffuso nelle reti irrigue dell'epoca, ossia il "regolatore" di Guglielmini (1690): una paratoia che, calata, crea un rigurgito a monte, trasformando la sezione del canale in un luce di fondo suscettibile di calcolo con la formula di Torricelli; a causa delle inesattezze di questi calcoli, l'idraulico milanese suggerisce in alternativa la misura diretta delle velocità con i tachimetri idraulici. Infine si 
occupa di migliorare i partitori asimmetrici, un manufatto assai inesatto, a causa della scala delle velocità trasversali, minime contro le pareti del canale e massime al centro (Fig. 7).
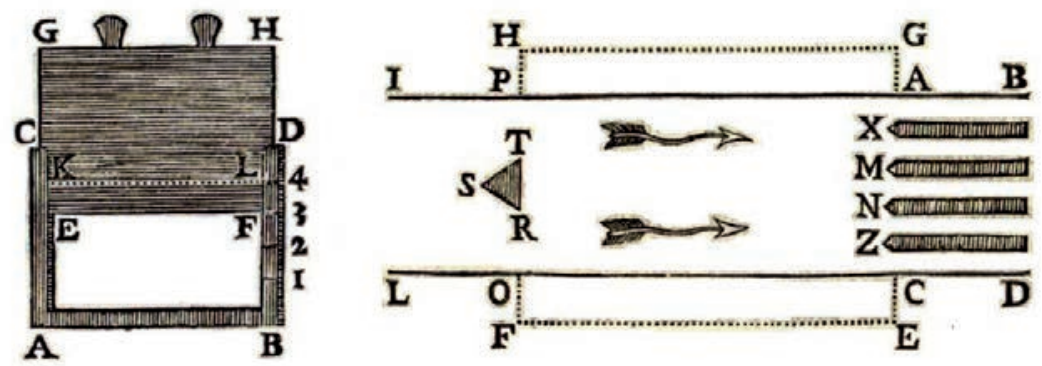

Fig. 7. A sinistra, il "regolatore" di Guglielmini, nella forma utilizzata dalle rogge milanesi alla metà del Settecento: sono segnate le altezze nell'unità di misura locale; la cateratta GH è calata dall'alto fin sotto il pelo dell'acqua, in modo tale da provocare un rigurgito a monte, che trasforma la sezione $A B E F$ in una luce di fondo, la cui portata è calcolata con la regola torricelliana; a causa dell'inesattezza di tale dispositivo, Lecchi

preferisce misurare direttamente le velocità puntuali con i tachimetri idraulici, deducendone la portata. A destra, un partitore con le modifiche suggerite da Lecchi per correggere lo squilibrio delle resistenze, maggiori nei canali laterali e minori in quello centrale (da Lecchi, 1765).

Contemporaneo di Lecchi è il barnabita milanese Paolo Frisi, uomo di punta dell'illuminismo, con carattere ed orientamento assai diverso, più teorico che pratico, portato a guardare con sufficienza le pratiche "volgari" degli ingegneri, facile ad assumere posizioni radicali e talvolta poco equilibrate. Sul tema della dispensa delle acque la sua posizione è l'esatto contrario di quella di Lecchi: questi, partendo dall'eccellenza (in termini relativi) del sistema milanese, propone una riforma, con criteri realistici e graduali, soprattutto degli altri sistemi più arretrati; quello invece, nel suo trattato di idraulica (1777), sferra un duro attacco proprio al sistema milanese, a cui dedica il capitolo intitolato Del riparto, delle regole, e degli errori delle bocche d'irrigazione, principalmente del Milanese.

In rappresentanza degli ingegneri idraulici milanesi, a Frisi risponde Francesco Bernardino Ferrari (1779). Il duello tra i due contendenti, il professore e l'ingegnere, vede la vittoria del secondo, il quale ha buon gioco nel dimostrare che l'edificio magistrale milanese è una macchina diversa, più complessa e più precisa di quella descritta 
dal primo, forte nella teoria ma debole nella pratica. Talvolta i lumi non sono sufficienti per evitare cantonate (Fig. 8).
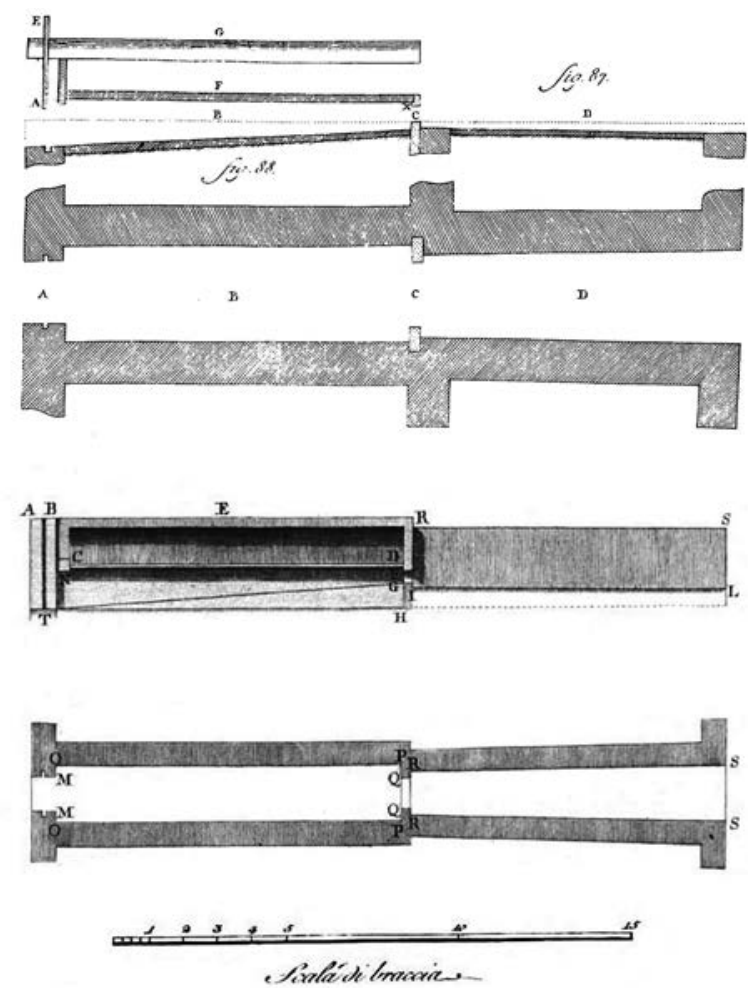

Fig. 8. In alto, l'edificio magistrale milanese nella rappresentazione di Paolo Frisi (1777); in basso, in quella di Francesco Bernardino Ferrari (1779).

In senso più profondo, il duello tra Ferrari e Frisi è quello tra Lecchi e Frisi, ossia tra due forme di riformismo, rispettivamente moderato e radicale. La posizione di Frisi potrebbe essere intellettualmente interessante, ma sbaglia i toni, non è tecnicamente ben argomentata $\mathrm{e}$ soprattutto non è una strategia: se lo fosse, dovrebbe evidenziare innanzitutto il divario esistente tra il sistema di irrigazione milanese (o meglio del Ducato di Milano) e il resto del Paese. Il problema esiste ed è importante per l'Italia e quindi il confronto è destinato a riproporsi qualche decennio dopo, con protagonisti diversi (Brunacci, Tadini, Romagnosi) ed esiti più fecondi. 


\section{IL DIBATTITO RIFORMISTA SULLA MISURA E LA DISPENSA DELLE ACQUE NEL REGNO NAPOLEONICO}

Nel 1813 la Società Italiana delle Scienze (detta dei Quaranta), fondata da Anton Mario Lorgna nel 1781 (un'iniziativa giustamente considerata prerisorgimentale, a cui partecipano molti scienziati idraulici!) indice un concorso per la soluzione del seguente quesito: Quale tra le pratiche usate in Italia per la dispensa delle acque è la più convenevole, e quali precauzioni ed artifici dovrebbero aggiungersi per interamente perfezionarla riducendo le antiche alle nuove misure metriche. Il concorso va collegato al tentativo del Regno d'Italia napoleonico di ricondurre ad unità i diversi sistemi di misura e dispensa delle acque in uso da secoli nelle varie province, adottando il sistema metrico decimale introdotto dalla rivoluzione francese. Il concorso è vinto nel 1814 dal toscano Vincenzo Brunacci (docente all'Università di Pavia) che prevale sul bergamasco Antonio Tadini (ispettore del Servizio "Acque e Strade" del Regno), con una coda polemica. Tadini, amareggiato, chiede la restituzione del suo manoscritto e si sente rispondere che è stato smarrito; furibondo e sospettando un complotto ai suoi danni, il coriaceo idraulico bergamasco ricompone lo scritto a memoria, per salvarlo dall'eterna oblivione e lo arricchisce con puntiglio, pubblicandolo con un commento polemico (1816). Vediamo ora le due posizioni a confronto, che possono essere definite rispettivamente di riformismo moderato e radicale.

Il documento di Vincenzo Brunacci (1814) è interessante dal punto di vista storico, perché fa un bilancio generale della situazione italiana all'inizio dell'Ottocento, concentrandosi soprattutto sull'Italia settentrionale. Al livello più basso, ma percentualmente limitato, troviamo tutte quelle costumanze, regolamenti, o concessioni, in virtù delle quali si estraggono acque dai fiumi, dai laghi, dai canali, siano essi di pubblica proprietà o di privata, senza che sia fatto alcun riflesso sulla quantità delle acque derivate. Un po' più numerose sono le pratiche in cui appare qualche indicazione sulla quantità dell'acqua, peraltro insufficiente per difetto di cognizioni idrauliche. E' ancora sporadicamente presente l'antico uso di proporzionare le portate erogate all'ampiezza delle luci dispensatrici, senza tener conto della velocità. In altri casi si prende per unità di misura la quantità d'acqua necessaria a mandare un mulino (chiamata macina d'acqua) o a girare una ruota (chiamata rota d'acqua) e si vendono due, tre, ecc. macine o ruote d'acqua, 
senza che sia stabilito quale sia precisamente questa quantità unitaria e come si possa concretamente estrarre. In altri casi ancora si prende come unità di misura la quantità d'acqua necessaria ad irrigare un campo di una data ampiezza, senza precisare la qualità della coltura ed il tipo di terreno. Tutte queste pratiche sono escluse dal confronto, perchè infatti non vi è in oggi fontaniere o altro custode di acque, che non ne conosca l'inesattezza.

Delimitato per esclusione il campo della ricerca, Brunacci lo caratterizza in positivo. Si tratta di confrontare tra loro quelle pratiche più avanzate per la misura delle acque, che sono state concepite dagli ingegneri e sancite dai governi sulla base della scienza idraulica, per identificare quella ottimale con criteri giuridici (esistenza di regole formali approvate dall'Autorità competente) e poi tecnici. Egli distingue tre categorie di manufatti per la dispensa delle acque: 1. luci libere (non regolate), aperte nella sponda del corpo d'acqua dispensatore, 2. luci regolate con cateratte, aperte nella sponda, 3 . luci modellate, costruite nello stesso canale derivato (edifici magistrali), che conservano sul modulo un battente dato. L'analisi si concentra su quattro pratiche in uso nel Mantovano - Veronese, nel Novarese, nel Cremonese e nel Milanese, che si collocano ai primi posti della graduatoria, con la netta vittoria dei due moduli (tra loro storicamente in competizione) messi a punto, nella seconda metà del Cinquecento, nel Ducato di Milano: la pratica Milanese prevale, ma di poco, su quella Cremonese. Nelle parole di Brunacci traspare chiaramente un forte apprezzamento, con parole come istupendissimo ritrovato, bellissima proprietà di tali edifizj delle bocche d'irrigazione, facil costruttura e mantenimento, ecc.

Si noti che le altre province del vecchio Ducato di Milano (Pavia, Lodi, Crema) hanno adottato con poche modifiche uno dei due moduli dominanti, mentre a sua volta la parte occidentale del Piemonte (Novara) usa un modulo simile a quello milanese, ma senza regolamentazione ufficiale (Figg. 9 e 10).

Le conseguenze politiche della valutazione vincente di Brunacci, fatta propria dall'influente Società dei Quaranta, sono evidenti: l'intero sistema dei manufatti per la dispensa delle acque nel Regno d'Italia napoleonico dovrebbe essere uniformato al modello milanese. All'epoca Milano è anche la capitale del Regno e finisce quindi per proporsi come un modello centrale a cui dovrebbero guardare le altre Province; che queste siano d'accordo non è certo: basti pensare che, nello stesso Ducato di Milano, sono convissuti per secoli due diversi e rivali modelli 
d'edificio magistrale, il Milanese e il Cremonese e che la provincia intermedia del Lodigiano ha pensato bene di adottare un modello con elementi dell'uno e dell'altro, al fine di evitare l'omologazione. Nella storia l'eccellenza italiana si mescola sempre alla difficoltà di fare squadra. In ogni caso, la situazione di isolamento di Milano, riscontrata nel Seicento e nel primo Settecento, appare completamente superata.

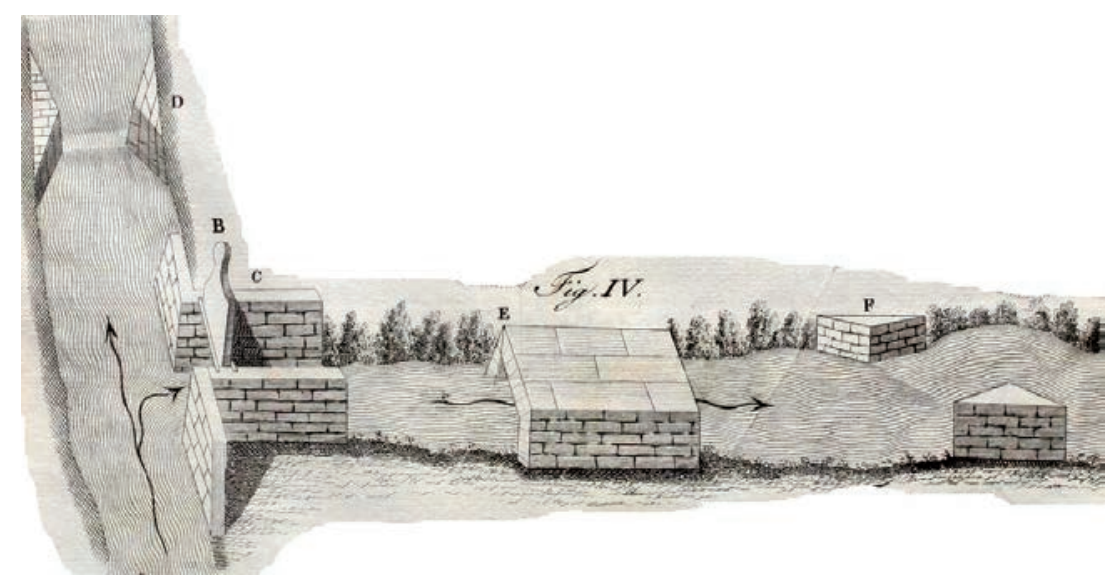

Fig. 9. L'edificio magistrale cremonese, con l'incile, la tromba con la bocca modellata e due sostegni a valle, detti briglia e scagno, quest'ultimo fuori figura (da Brunacci, 1814).

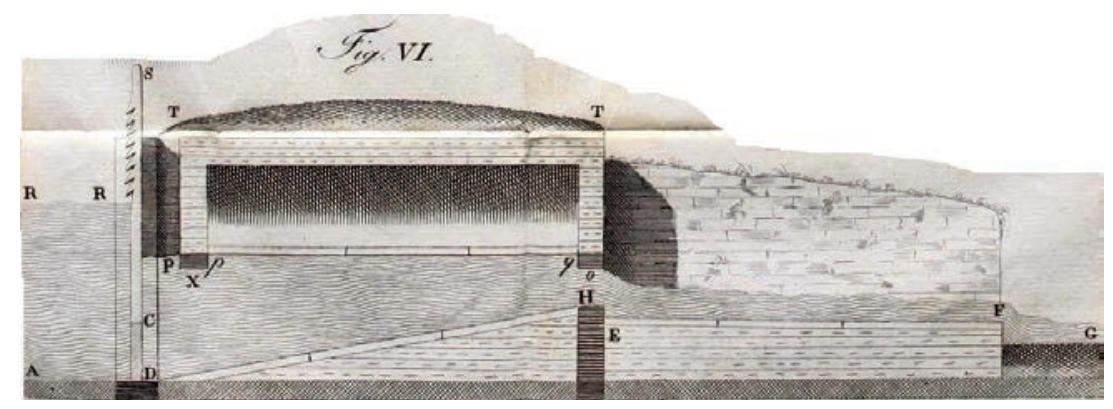

Fig. 10. L'edificio magistrale milanese, con la paratora, la tromba coperta, il cielo morto e la tromba scoperta (da Brunacci, 1814).

Veniamo ora alla posizione d'Antonio Tadini (1816). Dal punto di vista tecnico - operativo, il suo testo evidenzia una linea di riforma radicale, in netta opposizione a quella moderata di Brunacci. Egli, infatti, 
ritenendo superati tutti i sistemi esistenti per la misura e la dispensa delle acque, alla luce delle nuove conoscenze propone e sperimenta modelli alternativi, che dovrebbero essere subito messi in atto: i regolatori a piena doccia e a cateratta libera e il partitore delle acque a cascata. Di conseguenza, non è interessato a sviluppare un confronto analitico tra le varie pratiche in uso per la dispensa delle acque, ma si limita sostanzialmente a criticare l'edificio magistrale milanese, con pochi cenni agli altri sistemi. La posizione di Brunacci prevarrà fino alla metà dell'Ottocento (v. Carlo Possenti, 1858), mentre quella di Tadini si affermerà successivamente (v. Domenico Turazza, 1867).

Tadini riassume in termini efficaci l'impressionante divario che si riscontra in Italia tra l'area più avanzata, sull'asse Novara - Milano Cremona, con agricoltura intensiva che valorizza l'acqua per uso irriguo e il resto del Paese, che la spreca. Le sue parole contengono un implicito rimprovero alla scienza italiana: se nell'area del vecchio Ducato di Milano è presente da secoli un sistema avanzato che pratica l'irrigazione, e il resto del Paese la pratica male o la trascura, è anche perché questo tema è stato a lungo marginale nel dibattito scientifico e tecnico apertosi in Italia dopo il trattato di Castelli pubblicato nel 1628; esso merita la medesima attenzione riservata alle sistemazioni fluviali ed alla bonifica, i classici temi in cui eccelle la scuola idraulica italiana, in cui peraltro anche Tadini orgogliosamente si riconosce (si veda la posizione assunta contro le ingerenze dell'ingegnere di fiducia di Napoleone, Gaspard de Prony).

A prescindere dagli aspetti strettamente tecnico - scientifici, che appaiono più solidi di quelli esposti da Frisi, Tadini esprime una linea di riformismo radicale molto ben argomentata e convincente: non è più possibile tollerare il sistema dell' "oncia d'acqua", che non esprime la portata come volume d'acqua erogato nell'unità di tempo. Tutti i manufatti per la dispensa delle acque, anche i più avanzati, sono figli di una tradizione anteriore al trattato di Castelli (1628), il quale introduce il concetto di velocità nel calcolo della portata, e hanno continuato a parlare una lingua precastelliana, che conosce la sezione delle bocche e l'altezza del battente, ma non i volumi nell'unità di tempo. Continuare ad usare le vecchie unità di misura, che non indicano valori assoluti ma relativi delle quantità d'acqua erogate, e conosciuti per consuetudine e pressappoco soltanto nelle realtà locali, è irrazionale e comporta uno spreco inaccettabile di risorse.

A differenza di uno stereotipo oggi diffuso sui ritardi italiani rispet- 
to ai paesi europei più avanzati, le riforme proposte da Tadini non hanno alcun modello straniero di riferimento, anzi egli è ben consapevole che nonostante le sue critiche - il sistema irriguo lombardo rimane all'avanguardia in Europa. Questa situazione permane nei decenni successivi ed è pacificamente riconosciuta non soltanto in Italia (v. Elia Lombardini, 1844), ma anche all'estero (v. Nadault De Buffon, 1843 - 1844). La riforma propugnata da Tadini è dunque una questione tutta italiana.

\section{LA RIFORMA GIURIDICA DELINEATA DA ROMAGNOSI}

Con il suo Trattato della condotta delle acque (1823), Giandomenico Romagnosi, un'eccezionale figura di giureconsulto con solide conoscenze scientifiche, da una parte integra sul piano giuridico e politico la riforma proposta da Tadini, ma dall'altra va oltre, in senso liberale.

Romagnosi definisce il concetto di bocca legale d'erogazione, intesa come quella per la quale si fa constare venire somministrata la giusta competenza senza nuocere a terzi. La denominazione è generica e comprende tutti quei modelli e costruzioni che possono certificare essere somministrata la giusta competenza. A suo avviso le bocche magistrali hanno un credito d'opinione, ma sarebbe veramente il colmo della stolidaggine pensare che fuori delle cosi dette bocche magistrali non vi sia salute, ossia sicurezza d'erogazione.

Osserviamo che il principio affermato da Romagnosi vale anche per il regolatore di Tadini e per tutti gli altri manufatti e dispositivi inventati dagli ingegneri. Egli ama la scienza ma diffida degli scienziati e degli ingegneri, troppo inclini ad infatuarsi di un solo modello, da generalizzare attraverso una legislazione tecnica rigida, basata non tanto su principi generali di razionalità ed equità, per i quali egli stesso si batte, quanto sul parere e il progetto di un solo uomo, fallibile ancorché illuminato. Possiamo solo immaginare il disgusto, che proverebbe oggi Romagnosi alla lettura della dettagliatissima normativa tecnica moderna, elaborata in ogni campo dall'Unione Europea.

Notevole è la posizione critica assunta da Romagnosi nei confronti delle contraddizioni e dei ritardi della scienza e della tecnica, chiamate ad assistere il diritto nella valutazione se, ed entro quali limiti, le bocche d'irrigazione attuali possano essere considerate fedeli e quindi legali. Come avvocato, che è a contatto quotidiano con la realtà del territorio e gli interessi concreti in gioco nella distribuzione delle acque, egli 
sa che esiste una notevole differenza tra le teorie scientifiche e la pratica corrente. Il confronto tra gli esperti sulle bocche d'irrigazione, usate nei diversi paesi, avviene su basi teoriche e quindi insicure, che devono essere considerate con scetticismo. Gli stessi addetti ai lavori (i periti) sono consapevoli di queste lacune, ma coloro che potrebbero colmarle non se ne curano colpevolmente (Fig. 11).
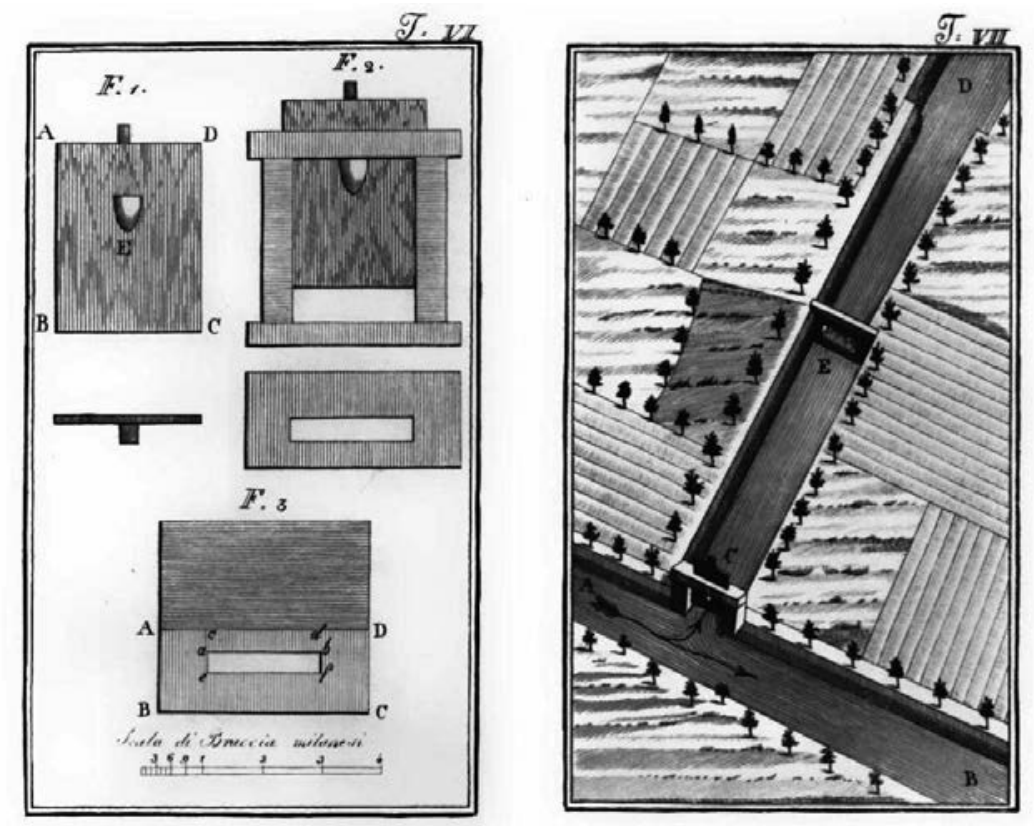

Fig. 11. A sinistra tipica cateratta in uso nel Milanese, all'inizio dell'Ottocento, per l'erogazione delle acque irrigue. A destra una bocca tassata per l'erogazione discontinua (turnata) dell'acqua: alzando la cateratta $C$, e tenendo l'occhio sul modulo E, il camparo può regolare il battente sul modulo stesso (da Romagnosi, 1823).

Dalle sue parole emerge un'evidente accusa d'inerzia, che investe il mondo scientifico e la pubblica amministrazione: perché non agiscono? La medesima accusa potrebbe essere estesa al settore giuridico: perché i giureconsulti, prima ancora di riformare il diritto, non interpretano in modo più aggiornato e più equo le stesse leggi vigenti, come egli ha suggerito? Il tema è complesso, perché va oltre la cultura, le leggi, la scienza e la tecnica e tocca il groviglio degli interessi e la stessa natura umana, come aveva già acutamente osservato Antonio Lecchi. 
9. UN ESTIMATORE FRANCESE DELLA TRADIZIONE IRRIGUA ITALIANA: NADAULT DE BUFFON

Una posizione che contrasta con quelle critiche di Tadini e Romagnosi per avvicinarsi a quella di Brunacci, è espressa da un ingegnere del Genio civile francese, a cui si riconosce il merito di aver fondato l'idraulica agraria francese: Benjamin Nadault de Buffon, il quale giunge in Italia, per ripetute visite di studio, quando ormai la gloriosa storia degli edifici magistrali sta percorrendo il tratto discendente della parabola. Egli non se ne accorge, perché abbagliato dall'eccellenza complessiva dello storico sistema irriguo milanese nel contesto europeo: la sua è una posizione rara dopo il Seicento, in un Paese come la Francia, ricco di primati e riluttante a riconoscere eccezioni alla sua grandezza, soprattutto in campi, che chiamano in causa l'operato dello Stato.

Di ritorno in patria, Nadault scrive un ponderoso trattato $\left(1^{\mathrm{a}} \mathrm{ed}\right.$. $1843-1844,2^{\text {a }}$ ed. 1861) dedicato al confronto tra il sistema irriguo dell'Italia settentrionale e quello del Midi francese, una regione simile per caratteristiche climatiche, ma molto arretrata nell'irrigazione, rispetto all'area d'eccellenza lombarda, a cui negli ultimi anni si è aggiunta quella piemontese, grazie non solo alla storica eredità milanese nel Novarese e nella Lomellina, ma anche alla modernità del recente Codice civile di Carlo Alberto (1837), redatto per la parte idraulica dal grande Giorgio Bidone e giudicato da Nadault (che ne ha parlato anche con il conte di Cavour!) la legge più avanzata del settore in Europa.

Il francese riconosce che, per la misura e la dispensa delle acque, i moduli hanno il gran vantaggio di far conoscere con esattezza la portata ricercata, senza ricorrere ad alcuna formula e ad alcun calcolo. E' stato il Ducato di Milano ad inventare i moduli e gli altri Paesi europei sono in ritardo di 5 - 6 secoli: la plupart des États d'Europe sont restés sur ce point, au degré le plus arriéré; c'est - à-dire, qu'on y concède encore l'usage des eaux courantes, exactement comme cela se faisait dans le Milanais, il y a cinq ou six siècles (cap. 16). Egli predilige l'edificio magistrale milanese rispetto a quello cremonese, che gli sembra inutilmente complicato e propone la sua estensione al Midi francese, salva la necessità di adattamento al sistema metrico - decimale: il y a lieu d'adopter, purement et simplement, le module milanais, car il est effectivement le plus exact des appareils de cette espèce, connus jusqu'à présent (Figg. 12-13). 


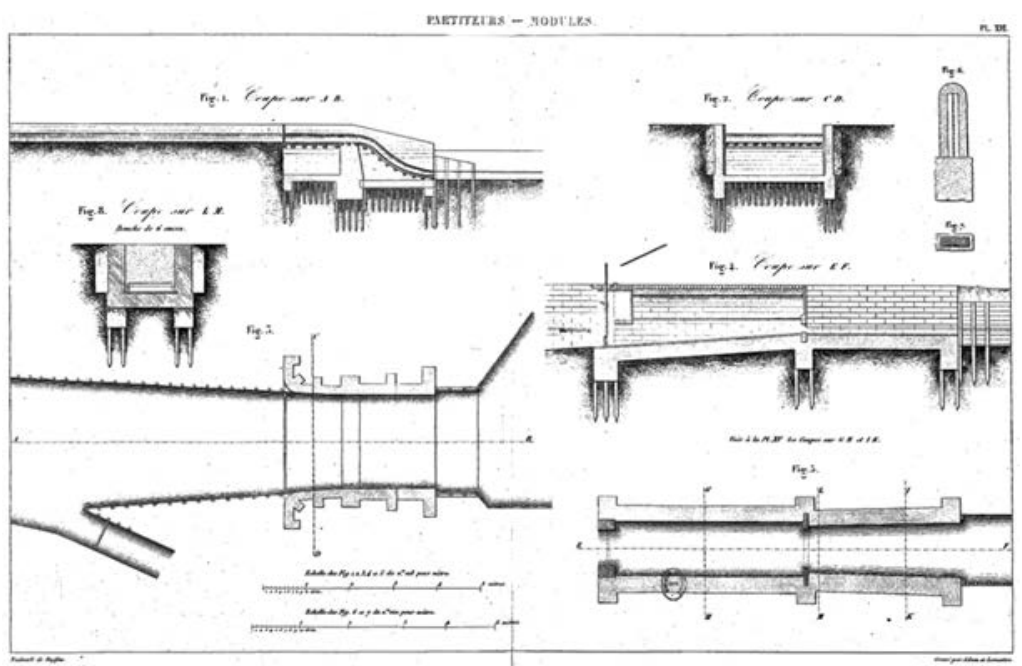

Fig. 12. Verso la metà dell'Ottocento, Benjamin Nadault de Buffon propone l'adozione nel Midi francese dell'edificio magistrale milanese. La figura illustra moduli e partitori in uso nell'Italia settentrionale (da Nadault de Buffon, 1861).

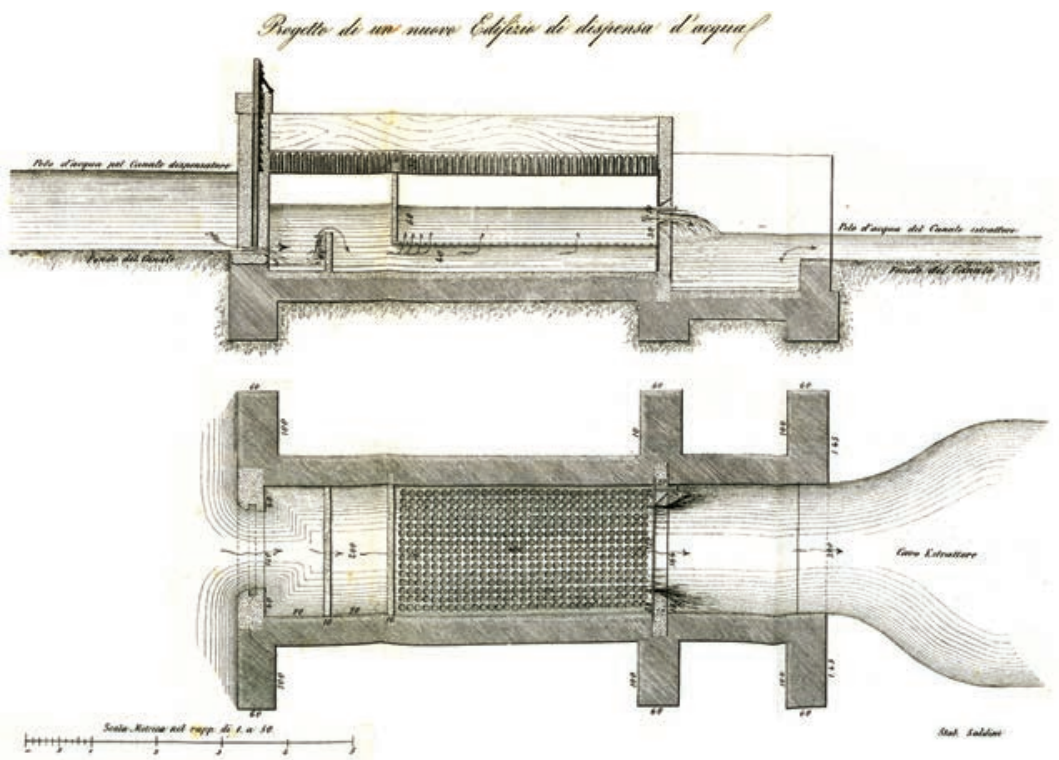

Fig. 13. Progetto migliorativo dell'edificio magistrale milanese, elaborato dall'ing. Carlo Possenti (1858). Rappresenta l'estremo tentativo di salvare lo storico manufatto introdotto nel Cinquecento per la dispensa delle acque nel Milanese. 
Notevoli sono anche i riconoscimenti del primato milanese sul piano giuridico - organizzativo. In Francia manca ancora l'istituto giuridico della servitù d'acquedotto, che consente al proprietario di un canale di farlo passare, a titolo oneroso, attraverso fondi altrui; nel Milanese questo istituto giuridico si afferma già nel XV secolo. Complessivamente, in tutte le epoche, questo Paese ha avuto leggi sulle acque correnti conformi alle più sane dottrine, che conciliano gli usi privati con l'interesse generale.

Pochi anni dopo (1852), anche l'Inghilterra riconosce il primato italiano, con il trattato Italian Irrigation di Richard Baird Smith, spedito in Italia dalla Compagnia delle Indie, impegnata nell'irrigazione del subcontinente indiano.

Contemporaneamente alla prima edizione del trattato di Buffon, ossia nel 1844, in occasione del Congresso degli scienziati tenutosi a Milano quell'anno, Carlo Cattaneo pubblica il suo libro Notizie naturali e civili della Lombardia. Nella prefazione vanta il primato europeo della Lombardia nell'irrigazione, con parole giustamente famose, che riassumono una gloriosa storia plurisecolare: Noi possiamo mostrare agli stranieri la nostra pianura tutta smossa e quasi rifatta dalle nostre mani... ..Abbiamo preso le acque dagli alvei profondi dei fiumi e dagli avvallamenti palustri, e le abbiamo diffuse sulle aride lande. La metà della nostra pianura, più di quattro mila chilometri, è dotata d'irrigazione; e vi si dirama per canali artefatti un volume d'acqua che si valuta a più di trenta milioni di metri cubici ogni giorno... Alla condutta di queste acque presiede un principio di diritto, tutto proprio del nostro paese, pel quale tutte le terre sono tenute a prestarsi questo vicendevole passaggio... senza intervento di principe, o decreto d'espropriazione... Gli ultimi scoli di tutte codeste acque sono muniti ai loro sbocchi di chiuse, che arrestano il rigorgo dei turgidi fiumi...

\section{BIBLIOGRAFIA}

BAIRD SMITH RICHARD, Italian Irrigation, being a report on the Agricultural Canals of Piedmont and Lombardy, addressed to the honorable the Court of directors of the East India Company, by R. Baird Smith, F.G.S., captain of engineers Bengal Presidency. 2. Vol. London. W. H. Allen and co.; Edinburgh, W. Blackwood and sons, 1852.

Barattieri Giovanni Battista, Architettura d'Acque, Piacenza, Stampa ducale di Giovanni Bazachi, 1656 -1663.

BRUNACCI VinCENZO, Memoria sulla dispensa delle acque (1814), in Nuova raccolta d'au- 
tori italiani che trattano del moto dell'acque, Tomo VI, Bologna, Tipografia Francesco Cardinali, 1829.

BRuschetTi Giuseppe, Storia dei progetti e delle opere per l'irrigazione del Milanese, Lugano, Giuseppe Ruggia, 1834.

CABEO NiCCOLÒ, De mensuratione aquarum decurrentium, in Philosophia experimentalis, sive In quatuor libros meteorologicorum Aristotelis commentaria et quaestiones (Liber primus, Textus 60), Romae, Typis haeredum Francisci Corbelletti, 1646.

Castelli Benedetto, Della misura dell'acque correnti, Libro I, Roma, Stamparia Camerale, 1628; Libro II, Bologna, Dozza, 1660, (postumo).

Di Fidio Mario - GANDOlfi Claudio, La lingua delle acque, in Biblioteca Idraulica Italiana, Milano, Fondazione Biblioteca Europea d'Informazione e Cultura, 2013.

Di Fidio Mario - GANDOlfi Claudio, Idrometria, in Biblioteca Idraulica Italiana, Milano, Fondazione Biblioteca Europea d'Informazione e Cultura, 2014.

FERRARI FranCESCO BERNARDINO, Lettera al signor conte don Gaetano di Rogendorf sulle bocche che estraggono acqua dai navigli, o sia canali navigabili di Milano (1779), in Raccolta d'autori italiani che trattano del moto dell'acque, Tomo IV, Bologna, Tipografia di Jacopo Marsigli, 1824.

FRISI PAOlO, Istituzioni di meccanica, d'idrostatica, d'idrometria e dell'architettura statica, e idraulica, ad uso della regia scuola eretta in Milano per gli architetti, e per gli ingegneri, Milano, Giuseppe Galeazzi Regio Stampatore, 1777.

Frontinus SeXtus Iulius, De aquaeductibus urbis Romae commentarius, antiqua fidei restitutus, atque explicatus opera et studio Ioannis Poleni, Patavii, apud Ioannem Manfrè, 1722.

Guglielmini Domenico, Aquarum fluentium mensura nova methodo inquisita, Bononiae, ex Typographia Pisariana, 1690.

Guglielmini Domenico, Della natura de' fiumi : trattato fisico-matematico, Bologna, per gl'eredi d'Antonio Pisarri, 1697.

LECCHI ANTONIO, Idrostatica esaminata ne'suoi principi e stabilita nelle sue regole della misura dell'acque correnti dal P. Antonio Lecchi della Compagnia di Gesù, matematico delle LL. MM. II., Milano, nella Stamperia di Giuseppe Marelli, 1765.

LEONARDO Da Vinci, Del moto e misura dell'acqua, Bologna, Francesco Cardinali, 1826.

LOMBARDINI Elia: Stato idrografico naturale e artificiale della Lombardia, in Carlo Cattaneo, Notizie naturali e civili della Lombardia, Milano, G. Bernardoni, 1844.

LOMBARDINI ElIA: Dell'origine e del progresso della scienza idraulica nel Milanese ed in altre parti d'Italia, Memorie del R. Istituto Lombardo, t. VIII - Giornale dell'ing. arch. Milano, 1860.

MiCHELOTTI FRANCESCO DOMENICO, Sperimenti idraulici principalmente diretti a confermare la teorica e facilitare la pratica del misurare le acque correnti, Torino, Stamperia reale, 1767 - 1771.

Michelini Famiano: Trattato della direzione de' fiumi, nel quale si dimostrano da' suoi veri principi i modi piu sicuri e meno dispendiosi di riparare a' danni che sogliono farsi dall'acque, Firenze, Stamperia della stella, 1664.

Nadault De Buffon Benjamin: Des Canaux d'arrosage de l'Italie septentrionale dans 
leur rapports avec ceux du Midi de la France; traité des irrigations envisagées sous les divers points de vue de la production agricole, de la science bydraulique et de la législation, Paris, Carilian - Goeury et V. Dalmont, 1843 - 1844.

Nadault de Buffon Benjamin: Hydraulique agricole. Applications. Des canaux d'irrigation de l'Italie septentrionale envisagés sous les divers points de vue de la production agricole, de la science bydraulique et de la législation. $2^{\mathrm{e}}$ édition. Dunod, Paris, 1861.

POSSENTI CARLO, Sull'edifizio magistrale milanese per la dispensa delle acque d'irrigazione, Milano, Tipografia Bernardoni, 1858.

Romagnosi GiAndOMEnico: Della condotta delle acque secondo le vecchie, intermedie e vigenti legislazioni dei diversi Paesi d'Italia, Milano, Tipografia di commercio, 1823.

TAdini Antonio, Del movimento e della misura delle acque correnti, Milano, Tipografia Sonzogno, 1816.

TORRICELli Evangelista, De motu aquarum, in Opera geometrica, De motu gravium naturaliter descendentium et Projectorum libri duo, Liber secundus, Florentiae, de Typis Amatoris Maffei et Laurentij de Landis, 1644.

Turazza Domenico, Trattato d'Idrometria o d'Idraulica pratica, 2a ed., Padova, Tipografia editrice Sacchetto, 1867.

Venturoli GiusepPe, Elementi di meccanica e d'idraulica, Vol. II, $3^{\text {a }}$ edizione, Milano, Paolo Emilio Giusti, Milano 1818.

ZENDRINI BERNARDINO, Leggi e fenomeni, regolazioni ed usi delle acque correnti, Venezia, Giambatista Pasquali, 1741. 